\title{
ADVANCEMENT OF PHOTOIONIZATION AND PHOTODISSOCIATION RATES RELEVANT TO ASTROCHEMISTRY
}

\author{
S P TARAFDAR \\ Tata Institute of Fundarnental Research \\ Homi Bhabha Road \\ Bombay 400 005, INDIA
}

ABSTRACT: The advancement of photoionization and photodissociation rates relevant for interstellar diffuse and dense clouds, circumstellar medium, cometary coma and planetary atmosphere has been reviewed with a mention of uncertainties involved in these rates.

\section{INTRODUCTION}

One of the most importance processes governing the molecular abundances in astronomical scenario is the photodissociation and photoionization. The role played by these processes in determining the molecular abundances in cometary coma and in planetary atmosphere were realised as early as 1930 (cf. Chapman 1930, Wurm 1932) bringing the subject of photochemistry in the forefront of astronomical studies. In interstellar medium the importance of photodissociation and photoionization were appreciated as early as 1941 by Swings (1941) when he attempted to understand the relative abundances of newly discovered (Swing and Rosenfeld 1937, McKeller 1940, 1941, Douglas and Herzburg 1941) CH, CN and $\mathrm{CH}^{+}$molecules though the possibility of photodissociation of molecules in interstellar space was mentioned earlier by Eddington (1926). The importance of photodissociation and photoionization in circumstellar medium has been appreciated only recently (cf. Gold reich and Scoville 1976, Scalo and Slavsky 1980, Clegg et al 1983), though the importance of ionization of atoms and ions were realised very early (cf. Stromgren 1948). The progress on the determination of photoionization and photodissociation rates were, however, hindered due to the lack of our knowledge about radiation field in ultraviolet (i.e. $\lambda<3000 \AA$ ) where most of the photodissociating and photoinoizing thresholds lie. With the advent of space astronomy, the radiation fields in the UV spectral region for different astronomical scenario starting with solar values become available and the determination of photoionization and photodissociation rates was possible. Some of these early determinations have been reviewed by Van Dishoeck (1987) in the first Astrochemistry Symposium at Goa (cf. Vardya and Tarafdar 1987). Van Dishoeck (1987) has also discussed various processes which lead to photodissociation. A discussion on processes leading to photoionization and photodissociation of molecules can also be found in Duley and Williams (1984). Here I would like to summarize the advancement made since meeting at Goa on the determination of photorates relevant to different astronomical objects which are distinguished from the flux of 
unattenuated radiation $F(\lambda)\left(p h \mathrm{~cm}^{1} \mathrm{~s}^{-1}\right)$ used in the photorate expression:

$$
\Gamma=\int_{0}^{\lambda_{t h}} \sigma(\lambda) F(\lambda) e^{\tau} d \lambda, s^{-1},
$$

where $\Gamma\left(s^{-1}\right)$ is the photorate, $\sigma(\lambda)$ the wavelength $(\lambda)$ dependent cross section, $\lambda_{t k}$ the threshold wavelength and $\tau$ is the optical depth of the medium at wavelength $\lambda$.

\section{INTERSTELLAR MEDIUM}

2.1 Rates with interstellar Diffuse radiation:

As reported by Van Dishoech (1987), the first comprehensive photorates for interstellar diffuse clouds were given by Black and Dalgarno (1977). Prasad and Huntress (1980) included these photorates in their compilation of reaction rates adding a number of photorates on their own. The rates given by Black and Dalgarno (1977) and Prasad and Huntress (1980) used interstellar radiation field which is in agreement with the interstellar field at $1000 \AA$ given by Habing (1968) and has a $\lambda^{3}$ dependence for $\lambda \leq 3000 \AA$. For $\lambda \geq 3000 \AA$, the used radiation field was the diluted blackbody field proposed by Spitzer (1968). The radiation field assumed to be zero for $\lambda<912 \dot{A}$, as absorption by hydrogen atom will inhibit the presence of radiation field at wavelengths smaller than its threshold wavelength of $912 \dot{A}$. Van Dishoeck $(1987,1988)$ determined photorates for a number of species using more reliable cross sections and a radiation field, the wavelength dependence of which determined from impirical fit to the interstellar radiation field (Witt and Johnson 1973 , Jura 1974) and is given by the relation (Draine 1978):

$$
F(\lambda)=\frac{3.210 \times 10^{15}}{\lambda^{3}}-\frac{5.17 \times 10^{17}}{\lambda^{4}}+\frac{2.064 \times 10^{20}}{\lambda^{2}} \text { photons } \mathrm{cm}^{-2} \mathrm{~s}^{-1} \AA^{-1}
$$

for $\lambda \leq 2000 \AA$ and

$$
F(\lambda)=6.973 \times 10^{2} \lambda^{0.7} \text { photons } \mathrm{cm}^{-2} \mathrm{~s}^{-1} \AA^{-1}
$$

for $\lambda \geq 2000 \AA$. Fig. 1 compares the radiation field used by Black and Dalgarno (1977), that given by equations (2) and (3) and the observed and computed radiation field by different authors. Fig. 1 shows that the flux given by equations (2) and (3) passes through maximum available values of radiation field and gives photorates a factor 1.5 to 2 larger depending on the threshold wavelength of dissociation or ionization than that obtained with other values of the field. Another uncertain factor related to interstellar radiation field is its attenuation by dust and molecular hydrogen. Recently Van Dishoeck and Black (1988) have shown that the $\mathrm{H}_{2}$ and $\mathrm{HI}$ absorptions could reduce $\mathrm{CO}$ dissociation by a factor of 1.5 . The grain absorption, however, remains very uncertain, as it depends on unknown grain properties which may vary from cloud to cloud. Different authors have given prescription for the inclusion of the effect of dust attenuation.

The calculation of photoionization and photodissociation rates was extended to more and more complex molecules (cf. Herbst and Leung 1986) resulting a comprehensible table (Miller et al 1991) of photorates for 92 species with 134 channels. The accuracy of these rates, however, is not very good. Besides, the uncertainty associated with the interstellar 


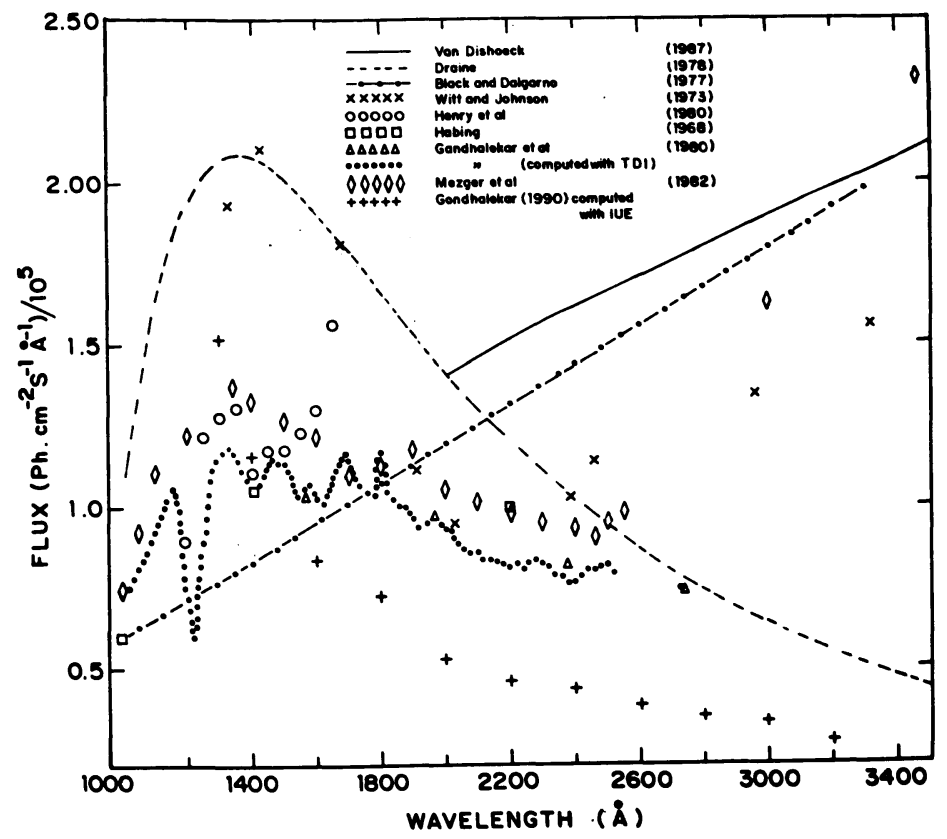

Fig 1: Comparison of observed and theoretically determined intersteilar radiation field with that used in photorate determination.

radiation field and its attenuation, the photodissociation and photoionization cross sections used for the determination of rates are very uncertain. To give an idea of uncertainty present, the photodissociation cross section are infered from molar extinction coefficient for $\mathrm{CH}_{3} \mathrm{OH}, \mathrm{C}_{2} \mathrm{H}_{5} \mathrm{OH}$ (Harrison et al 1959), $\mathrm{C}_{2} \mathrm{H}_{4} \mathrm{O}$ (Lake and Harrison 1959) and for $\mathrm{CH}_{3} \mathrm{OCH}_{3}$ (Harrison and Price 1959) and from low resolution absorption coefficients for $\mathrm{C}_{2} \mathrm{H}_{5} \mathrm{OH}$ (O gawa and Cook 1958). It is needless to mention that better cross sections for many molecules are needed.

\subsection{Photorates with cosmic ray induced emission from $\mathrm{H}_{2}$ :}

The interior of dense clouds is not completely devoid of ultraviolet photons as it was assumed conventionally. Prasad and Tarafdar (1983) have shown that the electrons produced by cosmic ray interaction with $\mathrm{H}_{2}, \mathrm{HI}$ and $\mathrm{He}$ can excite $\mathrm{H}_{2}$ molecules to its upper electronic states, the spontenuous decay of which act as source of UV-photons in the dense clouds. Prasad and Tarafdar (1983) estimated the flux of these photons in the interior of dense clouds and determined $\mathrm{CO}$ dissociation rates there. They came to the conclusion that dissociation rate of $\mathrm{CO}$ is large enough to give observed $\mathrm{CI} / \mathrm{CO}$ ratio in dense cloud in the steady state. Note that observed CI/CO ratio could be achieved in evolving models (Tarafdar et al 1985) and in time dependent models, if the life time of the cloud is not long enough to reach the steady state. Sternberg, Dalgarno and Lepp (1987) determined the UV-flux from cosmic ray induced $\mathrm{H}_{2}$ emission using Franck-Condon distri- 
bution for electron excitation to different vibrational levels of $B^{1} \Sigma_{z}^{+}$and $C^{1} \Pi_{w}$ upper electronic states and used the flux to determine the photodissociation rates for seven molecules $-\mathrm{CH}_{4}, \mathrm{C}_{2} \mathrm{H}_{2}, \mathrm{OCS}, \mathrm{NH}_{3}, \mathrm{HCN}, \mathrm{H}_{2} \mathrm{O}$ and $\mathrm{OH}$. Examining the effect of cosmic ray induced photons in dense clouds, they concluded that its presence will inhibit the formation of complex molecules. Gredel, Lepp and Dalgarno (1987) have determined the photodestruction rate of $\mathrm{CO}$ by cosmic ray induced photons as a function grain albedo, temperature and $\mathrm{CO} / \mathrm{H}_{2}$ ratio, as cosmic ray induced photons are line photons and photodestruction of $\mathrm{CO}$ is also by absorption of line photons. They concluded from their chemistry that $\mathrm{CI} / \mathrm{CO}$ ratio depends on the abundance, the rational population and the velocity distribution of CO. For a uniform cloud with $\mathrm{CO} / \mathrm{H}_{2}=1.5 \times 10^{-4}$ and $\mathrm{CO}$ rotational temperature of $30 \mathrm{~K}, C I / C O=5 \times 10^{-3}$ which is much larger than its value of $5 \times 10^{-6}$ without the presence of cosmic ray induced photons but smaller than the observed value of 0.1 . An agreement between theory and observation can be obtained, however, by postulating, a severe depletion of $\mathrm{C} / \mathrm{O}$ ratio and a differential velocity structure within the cloud. Gredel et al (1989) have improved the cosmic ray induced photon flux by taking the actual vibrational excitation distribution by electrons instead of Franck-Condon distribution and used this flux to determine photoionization and photodissociation rates for 37 species with 65 channels. The list of available photodestruction rates has been extended to 88 species with 193 channels by Tarafdar et al (1992) after determining the cosmic ray induced $\mathrm{H}_{2}$-emission flux independently including the effect of $\mathrm{H}_{2}$ and $\mathrm{CO}$ absorptions together with the absorption by grains. The photodestruction rates for common species in Gredel et al (1989) and Tarafdar et al (1992) agree farely well except for species having threshold near $1100 \AA$ or at shorter wavelength. For these species rates given by Tarafdar et al (1992) is smaller as $H_{2}$ absorption is more effective in the calculation of Tarafdar et al (1992) (Table 1). This discrepancy, however, is smaller than the uncertainty associated with the uncertain values of cross section mentioned before.

TABLE 1: comparison of photorates $\left(s^{-1}\right)$ given by Tarafdar et al (1992) and Gredel et al (1989) for species with threshhold wavelength near $1100 \AA$.

\begin{tabular}{ccllll}
\hline Species & Product & \multicolumn{2}{c}{ With $\mathrm{H}_{2}$-absorption } & \multicolumn{2}{c}{ Without $\mathrm{H}_{2}$-absorption } \\
& & Gradel et al & Present & Gredel et al & Present \\
\hline $\mathrm{CN}$ & $\mathrm{C}+\mathrm{N}$ & $1.8-13$ & $1.9-14$ & $2.0-13$ & $6.0-14$ \\
$\mathrm{O}_{2}$ & $\mathrm{O}_{2}^{+}+e$ & $1.8-15$ & $4.7-16$ & $2.3-15$ & $1.7-15$ \\
$\mathrm{Hcl}$ & $\mathrm{H}+\mathrm{Cl}$ & $9.0-15$ & $3.0-14$ & $1.2-14$ & $3.4-14$ \\
$\mathrm{NH}_{2}$ & $\mathrm{NH}+\mathrm{H}$ & $1.6-15$ & $5.8-15$ & $1.6-15$ & $6.3-15$ \\
$\mathrm{CH}_{3} \mathrm{OH}$ & $\mathrm{H}_{2} \mathrm{CO}+\mathrm{H}_{2}$ & $6.3-14$ & $4.0-13$ & $6.0-14$ & $4.0-13$ \\
$\mathrm{C}_{2} \mathrm{H}_{2}$ & $\mathrm{C}_{2} \mathrm{H}_{2}^{+}+e$ & $2.6-14$ & $6.3-15$ & $2.4-14$ & $1.2-14$ \\
& $\mathrm{C}_{2} \mathrm{H}+\mathrm{H}$ & $1.0-13$ & $2.3-14$ & $1.0-13$ & $2.4-14$ \\
$\mathrm{C}_{2} \mathrm{H}_{3} \mathrm{~N}$ & $\mathrm{C}_{2} \mathrm{H}_{3} \mathrm{~N}^{+}+\epsilon$ & $4.5-14$ & $4.6-15$ & $3.8-14$ & $1.9-14$ \\
$\mathrm{C}_{2} \mathrm{H}_{4}$ & $\mathrm{C}_{2} \mathrm{H}_{4}^{+}+e$ & $1.5-14$ & $3.1-15$ & $1.4-14$ & $6.3-15$ \\
$\mathrm{C}_{2} \mathrm{H}_{4} \mathrm{O}$ & $\mathrm{CH}_{3}+\mathrm{CHO}$ & $1.0-14$ & $2.8-15$ & $1.1-14$ & $3.0-15$ \\
& $\mathrm{CH}_{4}+\mathrm{CO}$ & $1.0-14$ & $3.2-15$ & $1.1-14$ & $3.4-15$ \\
\hline
\end{tabular}

$* a-n$ imply $a \times 10^{-n}$.

2.3 Photodissociation by photons from decay of Dark Matters:

Interior of dense clouds could have another source of photons - the decay of all pervading dark matters. Sciama (1990a) has shown that the source function $\varphi$ of photons arising from 
decay of dark matter (neutrinos) of density $\left(N_{d}\right) 5 \times 10^{7} \mathrm{~cm}^{-3}$ (Salucci and Sciama 1990) and life time $\tau$ of $1.5 \times 10^{23} \mathrm{~s}$ (Sciama 1990b) is given by

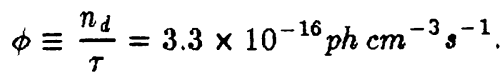

Sciama (1990b) has also argued that the photons arising from the decay of dark matters (DDM-photons) can have energy of 13.8ev. The observations were, however, not able to support the existence of such photons in cluster of galaxies (cf. Dadidson et al 1991, Fabian et al 1991). Certain loopholes in the observations need to be plugged before their existence is overruled (cf. Hogan 1991, Davidson et al 1991). Keeping the need of further proof in support or in against the possibility of the existence of DDM-photons Tarafdar (1991) determined the photon flux inside dense clouds considering absorption by HI and grains. The photodissociation rate of $\mathrm{CO}$ was calculated and the effect of such photons on $\mathrm{CI} / \mathrm{CO}$ ratio were examined. It was shown that if such DDM-photons exists, they can photodissociate $\mathrm{CO}$ to give the observed $\mathrm{CI} / \mathrm{CO}$ ratio.

\section{PHOTODISSOCIATION RATES FOR CIRCUMSTELLAR MEDIUM:}

The photoionization and photodissociation in circumstellar medium could take place due to stellar or interstellar radiation fields. In the circumstellar medium of cool stars like evolved high mass or newly formed low mass objects, the stellar radiation field in UV has low value so that the photoionization and photodissociation in circumstellar medium is by the interstellar radiation field with proper attenuation (cf. Glassgold, Huggins and Langer 1985; Omont 1986; Glassgold, Lucas and Omont 1986; Nejad and Miller 1987; Glassgold et al 1987; Mamon, Glassgold and Omont 1987; Mamon, Glassgold and Huggins 1988; Nejad and Miller 1988; Beiging and Nguyen-Quang-Rieu 1989; Nercession et al 1989; TruongBach et al 1990 and Howe and Miller 1990). In some cool stars, the stellar radiation field could also be important (cf. Clegg et al 1983). The dissociation of molecules by cosmic ray induced photons may not be important as its time scale of $10^{13}-10^{15} \mathrm{~s}$ is much longer than the expansion time scale $\left(\sim 10^{11} s\right)$ of the circumstellar medium. However, in a static circumstellar medium the cosmic ray induced photons could play some limited role. The photodissociation and photoionization in circumstellar medium around hot stars $(O$ and B stars) are mainly by stellar photons, as the ultraviolet flux from these stars is large. However, the interstellar photoionization and photodissociation rates scaled by appropriate factor (Cf. Tielens and Hullenbach 1985) can be used, as the stellar emission spectra (Fig. 2) has similar spectral shape in UV as interstellar radiation field. The error introduced by this assumption may be as large as an order or more (See Fig. 2) depending on the molecules (i.e. its threshold wavelength) and the spectral type (the temperature) of the central star.

\section{PHOTOIONIZATION AND PHOTODISSOCIATION IN COMETARY COMA:}

For cometary coma, the photoionizaation and photodissociation are governed by solar radiation field in UV. The earlier available rates (cf. Jackson 1976a, b and Huebner and Carpenter 1979) have been improved by Swift and Mitchell (1981) and more recently by Huebner, Keady and Lyon (1991). Note that photorates could be different from comets to comets and also in the same comet depending on its solar approach velocity which can 


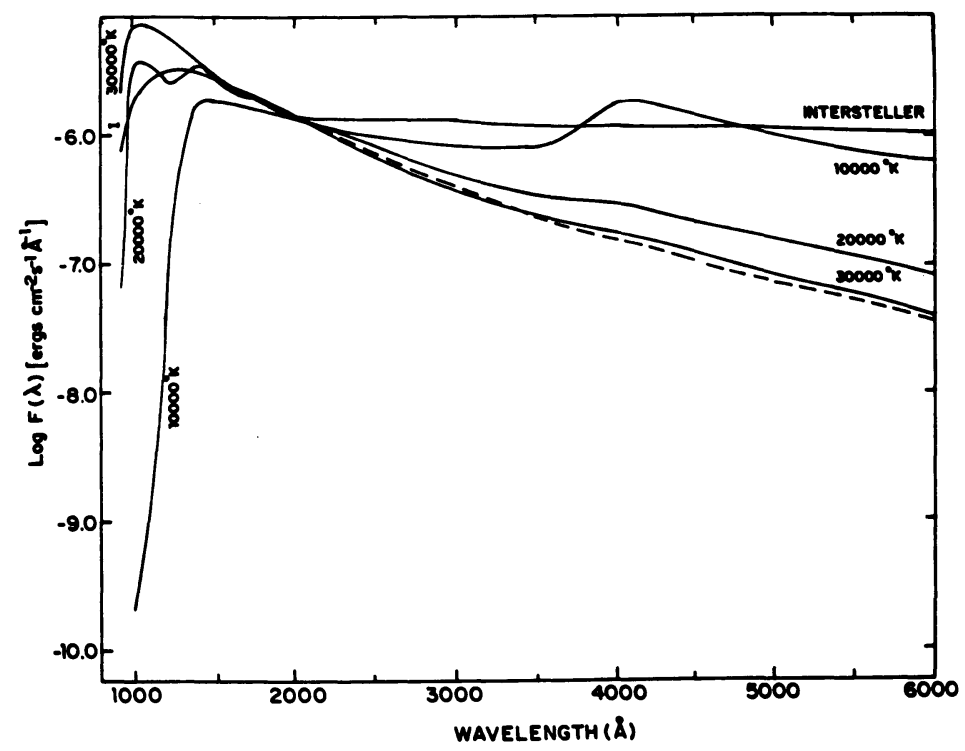

Fig. 2: Comparison of Interstellar radiation field used in photorate determination with stellar radiation fields (Kurucz 1779). Fields are normalised at $2000 \AA$.

shift the threshold wavelength of photoprocess resulting a variation of radiation field and the photorates, as solar radiation in UV varies significantly over small wavelength interval.

\section{PHOTOIONIZATION AND PHOTODISSOCIATION RATES FOR PLANETARY AND EARTHS ATMOSPHERE:}

The photodissociation and photoionization in planetary atmosphere is governed by solar ultraviolet radiation field as in cometary coma without the problem associated with velocity, as planetary orbits are circular. However, the photodissociation rates in planetary atmosphere has the inherent difficulty of accounting for the attenuation by atmospheric dust particles and the absorption by major constituent molecules. The photochemistry in different layers of Earth's atmosphere and in the atmospheres of planets has been discussed in a book edited by Levine (1985) who has also compiled the photodissociation rates in the appendix.

Acknowledgements: I would like to thank P. D. Singh and A. Dalgarno for enabling my participation in the symposium.

\section{REFERENCES:}

Black, J. and Dalgarno, A.: 1977, Astrophys. J. Suppl. 34, 405.

Bieging J. H. and Nguyen-Quang-Rieu: 1989, Astrophy. J. 343, L25. 
Chapman, S.: 1930, Mem. R. Meteorol. Soc. 3, 103.

Clegg R. E. S., Van Ijzendoorn, L. J. and Allamandola, L. J.: 1983, Mon. Not. R. Astr. Soc. $203,125$.

Davidson, A. F. et al: 1991, Nature, 351, 128.

Douglas, A. E. and Herzberg, G.: 1941, Astrophys. J. 94, 381.

Douglas, A. E. and Herzberg, G.: 1942, Can. J. Res. A20, 71.

Draine, B. T.: 1978, Astrophys. J. Suppl. 38, 595.

Duley, W. W. and Williams, D. A.: 1984, Interstellar Chemistry, Academic Press, London. Edington, A. S.: 1926, Proc. Roy. Soc. A111, 424.

Fabian, A. C., Naylor, T. and Sciama, D. W.: 1991, Mon. Not, R. Astr. Soc. 350, 461.

Glássgold, A. E., Huggins, P. J. and Langer, W. D.: Astrophys. J. 290, 615.

Glassgold, A. E., Lucas, R. and Omont, A.: 1986, Astron. Astrophys. 154, L12.

Glassgold, A. E., Mamon, G. A., Omont, A. and Lucas, R.: 1987, Astron. Astrophys. 180, 183.

Goldreich, P. and Scoville, J. M.: 1976, Astrophys. J. 205, 144.

Gondhalekar, P. M.: 1990, In The Galactic and Extragalactic Background Radiation. IAU Symp. No. 139, P49, eds. S. Bowyer and C. Leinert, Kluwer, Dordrecht.

Gondhalekar, P. M., Phillips, A. P. and Wilson R.: 1980, Astron. Astrophys. 85, 272.

Gredel, R., Lepp, S. and Dalgarno, A.: 1987, Astrophys. J. 323, L137.

Gredel, R., Lepp, S. and Dalgarno, A. and Herbst, E.: 1989, Astrophys. J. 347, 289.

Habing, H. J.: 1968, Bull. Astr. Inst. Neth. 19, 421.

Harrison, A. J. and Price, D. R. W.: 1959, J. Chem. Phys. 30, 357.

Harrison, A. J., Cederholm, B. J. and Terwilliger, M. A.: J. Chem. Phys. 30, 355.

Henry, R. C., Anderson, R. C. and Fastie, W. G.: 1980, Astrophys. J. 239, 859.

Herbst, E. and Leung, C. M.: 1986, Mon. Not. R. Astr. Soc. 226, 689.

Hogan, C. J.: 1991, Nature, 351, 96.

Howe, D. A. and Miller, T. J.: 1990, Mon. Not. R. Astr. Soc. 244, 444.

Huebner, W. F. and Carpenter, C. W.: 1979, Los Alamos Report LA8085-MS.

Huebner, W. F., Keady, J. and Lyon, S.: 1991, Astrophys. Space Sci. 1991 (in press).

Jackson, W. M.: 1976, J. Photochem. 5, 107.

Jackson, W. M.: 1976, In Study of Comets, IAU Coll. No. 25, p679, ed. B. Don.

Jura, M.: 1974, Astrophys. J. 191, 375.

Kurucz, R. L.: 1979, Astrophys. J. suppl. 40, 1.

Lake, J. S. and Harrison, A.: 1959, J. Chem. Phys. 28, 747.

Levine, J. S.: 1985, The Photochemistry of Atmospheres, Academic Press, New York.

Mamon, G. A., Glassgold, A. E. and Omont, A.: 1987, Astrophys. J. 323, 306.

Mamon, G. A., Glassgold, A. E. and Huggins, P. J.: 1988, Astrophys. J 328, 797.

McKellar, A.: 1940, Pub. Astr. Soc. Pac. 52, 307.

McKellar, A.: 1941, Pub. Dominion Astrophys. Observ. 7, 251.

Mezger, P. G., Mathis, J. S. and Panagia, N.: 1982, Astron. Astrophys. 105, 372.

Miller, T. J., Rawlings, J. M. C., Bennett, A., Brown, P. D. and Charnley, S. B.: 1991, Astron. Astrophys. Suppl. 87, 585.

Nejad, L. A. M. and Miller, T. J.: 1987, Astron. Astrophys. 183, 279.

Nejad, L. A. M. and Miller, T. J.: 1988, Mon. Not. R. Astr. Soc. 230, 79.

Nercession, E., Guillotean, S., Omont, A. and Benayoun, J. J.: 1989, Astron. Astrophys. $210,225$. 
Ogawa, M. and Cook, G. R.: 1958, J. Chem. Phys. $28,747$.

Omont, A.: 1986, in Circumstellar Matter, IAU Symp. No. 122, eds. I A ppenzeller and C. Jordan, P511, Reidel, Dordrecht.

Prasad, S. S. and Huntress, W. T. Jr.: 1980, Astrophys. J. Suppl. 43, 1.

Prasad, S. S. and Tarafdar, S. P.: 1983, Astrophys. J. 267, 603.

Scalo, J. M. and Slavsky, D. B.: 1980, Astrophys. J. 239, L 73.

Sciama, D. W.: 1990a, Astrophys. J. 364, 549.

Sciama, D. W.: 1990b, Phys. Rev. Lett. 65, 2839.

Spitzer, L.: 1968, Diffuse Matter in Space, Interscience, New York.

Stromgren, B.: 1948, Astrophys. J. 108, 242.

Sternberg, A., Dalgarno, A. and Lepp, S.: 1987, Astrophys. J. 320, 676.

Swing, P.: 1942, Astrophys. J. 95, 270.

Swing, P. and Rosenfeld, L.: 1937, Astrophys. J. 86, 483.

Swift, M. B. and Mitchell, G. F.: 1981, ICARUS 47, 412.

Tarafdar, S. P.: 1991, Mon. Not. R. Astr. Soc. 252, P55.

Tarafdar, S. P., Prasad, S. S., Huebner, W. F., Keady, J. and Lyon, S.: 1991, Astrophys. J. (to be published).

Tielens, A. G. G. M. and Hollenbach, D.: 1985, Astrophys. J. 291, 722.

Truong-Bach, Morris, D., Nguyen-Quang-Rieu and Deguchi, S.: 1990, Astron. Astrophys. $230,431$.

Van Dishoeck, E. F.: 1987, in Astrochemistry, IAU Symp. No. 120, P51, eds. M. S. Vardya and S. P. Tarafdar, D'Reidel, Dordrecht.

Van Dishoeck, E. F.: 1988, in Rate Coefficients in Astrochemistry, eds T. J. Miller and D. A. Williams, P49, Kluwer, Dord recht.

Van Dishoeck, E. F. and Black, J.: 1988, Astrophys. J. 334, 771.

Vardya, M. S. and Tarafdar, S. P.: 1987, Astrochemistry, IAU Symp. No. 120, D'Reidel, Dordrecht.

Witt, A. N. and Johnson, M. W.: 1973, Astrophys. J. 181, 363. 


\section{QUESTIONS AND ANSWERS}

P.Shapiro: Recently, space UV measurements have placed an upper limit on the flux near $13.6 \mathrm{eV}$ from a cluster of galaxies which is 10 times less than the prediction of Sciama's decaying neutrinos. Will this result not affect your conclusions?

S.P.Tarafdar: Recent space measurements (Davidson et al. 1991, Fabian et al. 1991) do put a limit on $13.7 \mathrm{eV}$ photon flux and if it is correct, then there should not be enough DDM-photons in ISM to affect its chemistry. However, there are some low photons in observations (e.g. Davidson et al. 1991 and Hogan et al. 1991) which puts some doubts on the observational interpretation.

V.Burdyuzha: Why you used the model hot dark matter?

S.P.Tarafdar: In hot dark matter model, neutrinos could be a possible constituent and Sciama (1990a) used this model to put values on its density, mass and lifetime.

P.Craine: (comment) If ther exist regions of the ISM which are convincing shown to be less than predicted by Sciama, then this can be used as an argument against the $27.8 \mathrm{eV}$.

S.P.Tarafdar: Yes, if we can show that DDM-photons arising from neutrinos of mass $27.8 \mathrm{eV}$ do some thing contrary to observation in ISM, it will argue against such neutrinos. Initially my aim was to try this. But $I$ found that neither ionization and heating rates nor the chemical effects of such photons are large enough to contradict any observation of ISM.

R.Gredel: Are there enough "Dark Matter Photons" to ionize atomic carbon and produce the observed abundances of [CII]?

S.P.Tarafdar: No, I do not think that DDM-photons can give observed $C^{+}$, if it is from dense part of the cloud. The flux of DDM-photons inside dense clouds is $\sim 65 /(1+$ $2 \times 10^{-4} n_{H}$ ) (Tarafdar, 1991) which will give $C^{+} / C O$ ratio of $0.16 / n\left(H_{2}\right)$ for $n_{H}<$ $5 \times 10^{3} \mathrm{~cm}^{-3}$ assuming formation of $C^{+}$by photoionization of $C$ with a cross section of $10^{-17} \mathrm{~cm}^{2}$ and destruction of $C^{+}$by reaction with $H_{2}$ with a rate of $4 \times 10^{-16} \mathrm{~cm}^{3} / \mathrm{s}$. The above $\mathrm{C}^{+} / \mathrm{CO}$ ratio is less than the observed value for any reasonable value of $n\left(\mathrm{H}_{2}\right)$. 